\title{
TRAP1 Regulates Autophagy in Lung Cancer Cells
}

Ines A. Barbosa ${ }^{1}$, Ignacio Vega-Naredo ${ }^{1,2}$, Rute Loureiro ${ }^{1}$, Ana F. Branco ${ }^{1}$, Rita Garcia ${ }^{1}$, Patricia M. Scott ${ }^{3}$, Paulo J. Oliveira ${ }^{1 *}$

${ }^{1}$ CNC-Center for Neuroscience and Cell Biology, University of Coimbra, UC Biotech Building, Lot 8A, Biocant Park, 3060-197 Cantanhede, Portugal

${ }^{2}$ Department of Morphology and Cell Biology, University of Oviedo, 33006 Oviedo, Spain.

${ }^{3}$ Department of Biomedical Sciences, University of Minnesota Medical School, Duluth Campus, MN, USA

\section{*Address correspondence to:}

CNC - Center for Neuroscience and Cell Biology

UC Biotech Building, University of Coimbra Lot 8A, Biocant Park, 3060-197 Cantanhede PORTUGAL

phone: +351-231-249-195, fax: +351-231-249-179

email: pauloliv@cnc.uc.pt

\section{Running title: TRAP-1 regulates autophagy and mitochondrial function in A549 cells}

Keywords: TRAP1; lung cancer; macroautophagy; apoptosis; cathepsin-B; autophagy

Word count: 3507 words, 5 images 


\begin{abstract}
Background: Expression of TRAP1, a member of the HSP90 chaperone family, has been implicated in tumor protective effects, based on differential mitochondrial localization and function.

Design: This work was designed to provide new insights on the pathways involved in TRAP1-provided cytoprotection on NSCLC. For this, TRAP1-depleted A549 human NSCLC cells and MRC-5 normal lung fibroblasts were produced using a siRNA approach and main cellular quality control mechanisms were investigated.

Results: TRAP1-depleted A549 cells displayed decreased cell viability likely due to impaired mitochondrial function including decreased ATP/AMP ratio, oxygen consumption and membrane potential, as well as increased apoptotic indicators. Furthermore, the negative impact of TRAP1 depletion on mitochondrial function was not observed in normal MRC-5 lung cells, which might be due to the differential intracellular localization of the chaperone in tumor versus normal cells. Additionally, A549 TRAP1-depleted cells showed increased autophagic flux. Functionally, autophagy inhibition resulted in decreased cell viability in both TRAP1-expressing and TRAP1-depleted tumor cells with minor effects on MRC-5 cells. Conversely, autophagy stimulation decreased cell viability of both A549 and MRC-5 TRAP1expressing cells while in A549 TRAP1-depleted cells, increased autophagy augmented viability.

Conclusions: Our results show that even though TRAP1 depletion affects both normal MRC5 and tumor A549 cell proliferation, inhibition of autophagy per se led to a decrease in tumor cell mass, while having a reduced effect on the normal cell line. The strategy of targeting TRAP1 in NSCLC shows future potential therapeutic applications.
\end{abstract}




\section{INTRODUCTION}

The chaperone gene Tumor Necrosis Factor Receptor-Associated Protein 1, TRAP1, is a member of the HSP90 family with predominantly mitochondrial localization, whose cytoprotective role has been widely documented [1-4]. Several studies suggest that TRAP1 preserves cellular function by protecting mitochondrial function by limiting the effects of oxidative stress by decreasing reactive oxygen species (ROS)-mediated oxidation [5] or by attenuating ROS production $[6,7]$, and by preserving mitochondrial membrane potential by limiting mitochondrial permeability transition pore (mPTP) opening through its interaction with HSP90 and cyclophilin D (CypD) [3, 8]. Recent studies identify additional cytoprotective roles for TRAP1 in cellular stress responses including the unfolded protein response to ER stress [9-11] and autophagy [12, 13]. Together, these effects seem to contribute for TRAP1 protective effect against apoptosis [5, 14]. These anti-apoptotic functions contribute to human oncogenesis. TRAP1 expression is increased in numerous human malignancies [15-17]. Functionally, inhibition of mitochondrial TRAP1 and related chaperones killed numerous tumor cells but not normal cells [8]. Anti-apoptotic functions of TRAP1 confer tumor cells resistance against different chemotherapeutic agents $[2,15]$. For these reasons, TRAP1 has been proposed as a potential biomarker and target for anticancer therapies. In this context, mitochondrial-targeted agents have been developed to selectively inhibit HSP90-like mitochondrial chaperones inducing cell death and reverting tumor cell multi-drug resistant phenotype [18].

It has been described that TRAP1 is overexpressed in a subset of lung adenocarcinomas $[19$, 20] and has been found to be more highly expressed in poor prognosis patients [21]. It was described that TRAP1 regulates proliferation, mitochondrial function, and has prognostic significance in non-small cell lung cancer (NSCLC) [21].

Despite the increased information regarding its cytoprotective functions, the characterization of TRAP1 antiapoptotic effects is far from being complete. The goal of this study is to better 
define the role of TRAP1 in the A549 human NSCLC cell line, specifically the relationship between its mitochondrial function and role in cellular stress responses. To this end TRAP1 expression was depleted in both A549 tumor and MRC-5 normal cell lines using siRNA and TRAP1 deficient cells were evaluated to determine TRAP1-dependent processes that contribute to cell viability.

\section{MATERIALS AND METHODS}

\section{Cell culture and siRNA transfection}

Human A549 lung cancer cells and MRC-5 normal lung fibroblasts were purchased from ATCC (LGC Standards, Middlesex, UK), and cultured in Iscove's Modified Dulbecco's Medium (IMDM, 12440-046; Gibco, Paisley, UK) supplemented with 5\% FBS (16000-044, Gibco) and antibiotic/antimycotic solution (A5955, Sigma; St. Quentin Fallavier, France), at $37^{\circ} \mathrm{C}$ in humidified air with $5 \% \mathrm{CO}_{2}$. Details on cell transfection protocols can be seen in the supplementary information (SI).

\section{Cell mass}

Cell mass was measured by the Sulforhodamine B (SRB) assay [22]. Complete experimental details can be seen in the SI.

\section{Western blotting analysis}

Details on Western blotting analyses can be found in detail in the supplementary material.

\section{Real-time RT-PCR}

The experimental procedure for RT-PCR can be found in detail in the SI.

Mitochondrial transmembrane electric potential $(\Delta \psi \mathrm{m})$ and mitochondrial superoxide anion 
Mitochondrial transmembrane electric potential $(\Delta \psi \mathrm{m})$ and mitochondrial superoxide anion were evaluated using fluorescent dyes, as described in the SI.

Mitochondrial permeability transition pore (mPTP) opening

Determination of mPTP opening was performed using the MitoProbe Transition Pore Assay

(M34153; Invitrogen) by co-loading with calcein-AM and $\mathrm{CoCl}_{2}$ as previously described

[23]. Further details are present in the supplementary materials and methods.

\section{Oxygen consumption and glycolytic fluxes}

Oxygen consumption rates (OCR) and extracellular acidification rates (ECAR) were measured using the Seahorse XF 96 Extracellular Flux Analyzer Technology (Seahorse Biosciences; North Billerica, MA, USA), as described in detail in the SI.

\section{Adenine nucleotide measurement (ATP/ADP/AMP)}

ATP, ADP and AMP levels were measured by HPLC as described in detail in the SI.

\section{Immunocytochemistry}

The protocol for immunocytochemistry is detailed in the SI.

\section{Mitochondrial morphology and lysosome content}

The experimental protocol for the evaluation of mitochondrial morphology and lysosomal content is described in detail in the SI.

\section{Enzymatic activities}

Activities of cysteine-proteinase cathepsin B, aspartate-proteinase cathepsin D, and caspases $3,-7$, and -9 were measured as described in detail in the SI.

\section{Live/dead assay}


Calcein-AM (C1430; Invitrogen) and Propidium Iodide (PI) (P4864; Sigma Aldrich) were used to determine the percentages of viable and dead cells. The detailed protocol can be found in the SI.

\section{Statistical analyses}

For statistical analysis of two means Student's $t$ test was used while for comparisons of more than two groups an analysis of variances (ANOVA) with matched-pairs was used instead. In both cases, data was checked for normality using the Kolmogorov-Smirnorv with DallalWilkinson-Lillie for correction and equality of variances using the F test or Bartletts's test in order to apply the statistical tests. Otherwise the respective non-parametric test was chosen: Mann-Whitney for $\mathrm{t}$ test and Friedman test for ANOVA.

In situations where one wanted to evaluate the effect of two variables a two-way ANOVA was applied following the same assumptions as mentioned earlier. Differences were considered significant at 5\% level and $\mathrm{p}$ value was categorized accordingly to their interval of confidence. For one- and two-way ANOVA, corrections for multiple comparisons were made using Bonferroni post-hoc test using predefined comparisons, to decrease both type I and type II errors.

\section{RESULTS}

\section{TRAP1 depletion caused decreased viability in A549 cells.}

We initially investigated by Western Blotting the amount of TRAP1 expressed in both A549 and MRC-5 cell lines in order to identify differential expression levels. This experiment showed that A549 lung cancer cells showed the highest expression of TRAP1 (Fig. S1A) To characterise the overall outcome of TRAP1 inhibition on mitochondrial physiology, a knockdown was performed in both A549 and MRC-5 cell lines using a siRNA construct (siTRAP1) (Fig. S1B).

In accordance with previous reports [21, 24], TRAP1 silencing resulted in a decrease in tumor cell proliferation rate within 24 hours post-seeding leading to a $62 \%$ reduction of A549 cell 
mass during 72 hours (Fig. S1C). Increased cell death contributes to decreased viability as calcein-AM and propidium iodide Live/Death assay confirmed that A549 TRAP1 silencing increases the percentage of dead cells (Fig. S1D). Interestingly, TRAP1 silencing resulted in a significant decrease in MRC-5 cell proliferation (Fig. S1C) leading to a drop of $49 \%$ in cell mass.

\section{TRAP1 silencing resulted in mitochondrial dysfunction}

As TRAP1 cytoprotective role has been mainly associated with its preferential mitochondrial localization within tumor cells [25] we examined the effect of TRAP1 depletion on mitochondrial function in A549 and MRC-5 cells. TRAP1 subcellular localization was evaluated through cell imaging by co-staining cells with antibodies against TRAP1 and the outer mitochondrial membrane marker TOM20. In agreement with the literature, immunofluorescence assays showed TRAP1 co-localization with TOM20 in A549 control cells (scrambled siRNA, siCTL) with a small amount of fluorescent signal dispersed throughout the cell, as previously demonstrated [26] (Fig. 1A). Interestingly, even though TRAP1 co-localization with TOM20 was also observed in MRC-5 cells, this mitochondrial chaperone seems to have a sparse and diffused distribution in these cells, instead of the expected major mitochondrial localization, suggesting TRAP1 preferentially localizes in extra-mitochondrial sites in MRC-5 cells (Fig. S3A). No alteration in TOM20 protein content was observed after TRAP1 silencing (Fig. S2A).

Overall A549 siTRAP1 cells bioenergetic capacity was decreased as indicated by decreased ATP/ADP ratio and adenylate energy charge (Fig. 1B), and decreased oxygen consumption, both basal (Fig. 1C) and in the presence of the mitochondrial uncoupler FCCP (Fig. 1D) although glycolytic rates remained unaltered upon TRAP1 silencing when indirectly inferred through the evaluation of extracellular acidification rate (ECAR) (Fig. S2B). Further evidence of disrupted electron transport chain was provided by increased Mitosox staining indicating increased superoxide accumulation in TRAP1 depleted cells (Fig. 1E). Finally, mitochondrial membrane potential was diminished as shown by decreased TMRM staining of TRAP1 
depleted cells (Fig. 1F). Conversely, our findings indicate that TRAP1 silencing does not lead to changes in MRC-5 oxidative stress levels and mitochondrial membrane potential (Fig. S3B).

Considering that mitochondrial depolarization together with decreased ATP production and inhibition of respiration constitute some of the later stage effects of mPTP opening, and since TRAP1 has been described to modulate mPTP by binding to and antagonizing CypD function conferring tumor cells protection against apoptosis [8], the open/closed conformation of the mPTP was measured. Surprisingly, TRAP1 depletion in A549 resulted in a more closed mPTP conformation when compared to siCTL cells (Fig. 1G) suggesting other factors may be contributing to the observed mitochondrial impairment, whereas in MRC-5 cells no changes were observed in $\mathrm{mPTP}$ opening status (Fig. S3D). The results observed for the lung tumor cell line were not due to defective CypD function in TRAP1 depleted cells as equivalent CypD protein content was observed (Fig. S2C) and cyclosporin A was effective in closing the mPTP (Fig. 1G). Thus, in these cells TRAP1 deficiency results in loss of mitochondrial membrane potential through a means other than promoting opening of the MPTP.

\section{TRAP1 depletion altered mitochondrial morphology}

Additionally, TRAP1 depletion in A549 cells was accompanied by alterations in mitochondrial morphology while no changes in organelle morphology were observed between the MRC-5 cell groups (Fig. S3E). Mitochondria were stained by Mitotracker Red (MTR) dye to delineate morphology and morphology was quantitated. The average area/perimeter ratio was employed as an index of mitochondrial interconnectivity and inverse circularity used to ascertain mitochondrial elongation [27]. By these measures siTRAP1 A549 cells displayed reduced elongation and interconnectivity, confirmed by staining with MTR (Fig. 2A). This was accompanied by an increase in dynamin-related protein 1 (DRP1) levels with no alterations of in expression of FIS1 or either of the mitochondrial fusion proteins, optic atrophy protein 1 (OPA1) and mitofusin-1 (MFN1), suggesting that altered morphology 
following TRAP1 silencing was due to enhanced mitochondrial fission (Fig. 2B). These observations, together with the fact that no alterations were found in the expression of the studied mitochondrial dynamics proteins in MRC-5 cells (Fig. S3F), highlight the role for TRAP1 in the maintenance of mitochondrial morphology, which would be more pertinent in cells under stress, such as in tumors.

\section{TRAP1 silencing altered A549 cell death machinery}

To determine how loss of mitochondrial membrane potential contributes to decreased cell viability and increased cell death, several apoptotic pathways were analyzed. Since A549 cells do not express BCL-2 [28], the expression of pro-apoptotic BAX and anti-apoptotic BCL-xL proteins was measured to determine mitochondrial permeability mediated apoptosis was enhanced. TRAP1 silencing in these cells induced a significant increase in BAX protein content along with a marked decrease of BCL-xL (Fig. 3A) suggesting the activation of intrinsic apoptosis. The BAX/BCL-xL ratio was of $2.10 \pm 0.18(n=6)$ for siTRAP1 cells and $0.79 \pm 0.03(\mathrm{n}=6)$ for siCTL cells $(\mathrm{p}<0.001)$, with the difference mainly due to decreased BCL$\mathrm{xL}$ expression. Therefore, TRAP1 seems to protect cells from mitochondrial apoptosis mainly through up-regulation of BCL-xL anti-apoptotic protein expression. Interestingly, the expression of BAX and BCL-2 proteins in MRC-5 cells showed a BAX/BCL-2 ratio of $1.06 \pm 0.07(\mathrm{n}=4)$ for siTRAP1 and $1.00 \pm 0.15(\mathrm{n}=4)$ for siCTL group $(\mathrm{p}>0.05)$, indicating that in these normal cells, TRAP1 does not alter the pro- anti-apoptotic protein balance (Fig. S3G).

Notwithstanding, TRAP1 silencing increased expression of key proteases involved in cell death. siTRAP1 cells showed a higher expression of pro-caspase-3 (Fig. 3B) in A549 cells and increased activity of Caspase 3/7 in both A549 and MRC-5 cells (Fig. 3C and S3G). Cathepsins D and B are involved in processes of lysosomal cell death being able to activate caspases cascade and directly cleave pro-caspase-3 [29-31]. Increased cathepsin B activity was observed in A549 cells (Fig. 3D), despite reduced expression of cathepsins D and B and no change in cathepsin D activity (Fig. 3E). 
To further delineate apoptosis pathways activated by TRAP1 silencing, we measured the activities of initiator caspases 8 and 9 (Fig. 3F) which are associated with extrinsic and intrinsic pathways, respectively. Our results argued against activation of classical apoptotic pathways after TRAP1 silencing since we did not find increases in initiator caspases 8 (extrinsic) and 9 (intrinsic) activities in siTRAP1 A549 cells (Fig. 3F). However, it is important to note a higher caspase 9 activity was observed in siTRAP1 MRC-5 cells (Fig. S3G).

Observations in the A549 cell line suggest the activation of alternative forms of cell death, possibly related with the development of acidic organelles and the activation of the lysosomal protease cathepsin B described above. Overall, our data suggests distinct forms of cell death pathways are activated as a result from TRAP1 depletion depending on the importance of this chaperone for mitochondrial maintenance in tumor vs normal lung cells.

\section{Autophagy is altered in TRAP1 depleted cells.}

Our results demonstrate mitochondrial damage with likely mitochondrial fission carried out by DRP1 in A549 TRAP1 depleted cells. As damaged mitochondria can be removed by autophagy we focused on the effect of TRAP1 on this process. Conversion of microtubuleassociated protein 1A/1B-light chain 3 (LC3-I, 18-kDa) to LC3-II (16-kDa) is an essential step in the formation of the autophagic vesicle. Thus, accumulation of LC3-II is considered the most definitive marker for autophagosomes [32]. Initial measurements of basal LC3-II levels show no significant difference between TRAP1 expressing and TRAP1-depleted cells (Fig. 4A). However, since LC3-II is subjected to lysosomal degradation during autophagy, LC3-II levels do not accurately reflect autophagy induction. To test if autophagic flux is increased in TRAP1depleted cells, LC3-II expression was analyzed in both siCTL and siTRAP1 A549 cells treated with Bafilomycin A1 (BafA1), a known inhibitor of the late phase of autophagy due to its effects in neutralizing acidic organelles [33]. Even though no differences were observed in non-treated siCTL and siTRAP1 cells, LC3-II content increased upon BafA1 treatment in both siCTL and siTRAP1 cells. However, LC3-II accumulation is 
significant and shows a greater fold change in siTRAP1 cells supporting increased autophagic flux after TRAP1 depletion (Fig. 4A). Although levels of several enzymes involved in autophagy are lower in TRAP1 depleted cells (Fig. 4B) further investigation provides evidence of increased autophagic flux. Disruption of autophagy often results in the accumulation of autophagy ubiquitin-binding adaptor p62/SQSTM1 (sequestosome-1) [34]. In support of increased autophagic flux, p62 protein levels in siTRAP1 A549 cells were significantly decreased (Fig. 4B). Moreover, TRAP1 silencing in MRC-5 led to decreased ATG12-ATG5 complex, LC3 and p62 protein levels, also indicating an increase in autophagy flux in these cells (Fig. S4A).

In addition, the area of lysotracker green labelled cellular organelles increased dramatically in siTRAP1 A549 cells, indicating an expansion of acidic organelles including the lysosome, the site of degradation of autophagic cargo (Fig. 4C). In addition, levels of LAMP2A protein, a lysosomal receptor involved in chaperone mediated autophagy (CMA) [35], are increased in TRAP1 depleted cells suggesting possible involvement of CMA (Fig. 4D). No differences were observed between siCTL and siTRAP1 MRC-5 cells for lysosomal content and CMA markers (Fig. S4A and B).

To evaluate the effect of TRAP1 silencing on ER stress and the unfolded protein response (UPR) in A549 cells, we measured the expression of several key chaperones that have been implicated in TRAP1 mediated alteration of the UPR- HSP90, Glucose-regulated protein 78 (GRP78) and GRP94. No alteration in the expression of these chaperones was detected, suggesting that TRAP1 silencing did not induce ER-stress and the consequent UPR in A549 cells (Fig. S2D).

\section{TRAP1 silencing altered the outcome of increased autophagy}


Autophagy plays a major role in maintaining cell quality control and homeostasis but under extreme conditions autophagic signaling can be recruited to activate cell death pathways. Thus, autophagy can act either as a tumor suppressor or as a tumor maintenance factor [36, 37]. To better understand the functional significance of the autophagic activation observed in siTRAP1 cells, transfected cells were treated with either the autophagy inducer rapamycin or the autophagy inhibitor 3-methyladenine (3-MA) for up to 72 hours and evaluated for cell viability. Inhibition of autophagy by inhibition of lysosomal acidification with 3-MA leads to decreased cell viability in both TRAP1-expressing and TRAP-depleted A549 cells indicating that autophagy promotes cell viability in these cells (Fig. 5A). Although similar observations were made for MRC-5 cells, the decrease in siCTL cell mass upon treatment with 3-MA is not as strong, leading to an overall decrease of only about $20 \%$ whereas this difference reaches a value of $400 \%$ for the correspondent A549 cell groups (siCTL vs siCTL treated with 3-MA) (Fig. 5A and Fig. S4C). In contrast, treatment with Rapamycin, which activates autophagy by inhibition of mammalian target of rapamycin (mTOR), showed contradictory results. As expected, activation of autophagy is protective for A549 TRAP1 depleted cells. However, Rapamycin treatment of TRAP1 expressing cells is severely deleterious to these cells in contrast with what is observed for MRC-5 cells (Fig. 5B). Induction of autophagy in MRC-5 cells resulted in a significant cell mass decrease in siCTL cells with no change observed for the TRAP1-depleted cells (Fig. S4C).

These results demonstrate that autophagy at endogenous levels is protective for TRAP1 expressing A549 cells. However, the effect of activation of autophagy above endogenous levels is context dependent and TRAP1 expression levels alter this context. Mechanistically, both increased endogenous autophagic flux and increased tolerance for induced autophagy in A549 TRAP1-depleted cells may be a consequence of mitochondrial dysfunction creating a greater need for autophagic mitophagy to remove damaged mitochondria which our results suggest might be specific to tumor cells. 
1

2

3

4

5

6

7

8

10

\section{DISCUSSION}

In agreement with previous studies [25], we found that TRAP1 co-localizes with mitochondria in A549 cells, based on co-localization with the outer-mitochondrial protein TOM20. In addition, TRAP1-depleted cells showed impairment of multiple parameters of mitochondrial function as previously reported including decreased $\Delta \Psi_{\mathrm{m}}$, increased ROS production, and lower oxygen consumption. The increased mitochondrial ROS production in TRAP1-depleted cells can trigger cell death by mPTP opening, which in turn results in $\Delta \Psi_{\mathrm{m}}$ loss. TRAP1, along with HSP90 mitochondrial pool and HSP60, have been described to physically interact with matrix CypD modulating its regulatory activity and resulting in mPTP inhibition $[3,8]$. Surprisingly, TRAP1-depletion resulted in a more closed mPTP conformation under basal conditions, which was the opposite effect to that observed in melanoma [38]. Our result was not due to loss of CypD expression or activity as CypD expression was intact and addition of cyclosporin-A resulted in an increase in mPTP closing. The discrepancy with previous work may reflect that these studies relied on extrapolation of mPTP status from analysis of $\Delta \Psi_{\mathrm{m}}$ alterations [8] or on evaluation of cell viability in the presence or absence of CsA [3]. These results suggest that TRAP1 can influence mitochondrial-mediated viability in A549 cells by means other than inhibition of CypD. Accumulation of depolarized mitochondria and oxidative damage has been associated with the occurrence of mitochondrial fusion and fission processes in an attempt to either restore homeostasis or eliminate mitochondria that are beyond repair [39]. Consistent with mitochondrial impairment, TRAP1 depletion has been reported to result in altered in mitochondrial morphology, including both fragmentation [40] and formation of tubules [41]. Here, TRAP1 silencing in tumor cell line resulted altered mitochondrial morphology characterized by the accumulation of smaller and rounder mitochondria and increased expression of the fission protein DRP1 without affecting expression of FIS1 or the fusion proteins MFN1 and OPA1.

Our results suggests that TRAP1 may differentially localize in normal (MRC-5) versus tumor (A549) cells which might explain why TRAP1 depletion in MRC-5 cells had no implications 
on mitochondrial physiology and morphology. Based on our observations, we hypothesized that TRAP1 preferentially localize to extramitochondrial sites in normal cells thus not having such an important role in mitochondrial homeostasis maintenance.

The process of mitochondrial fragmentation has been reported to occur almost simultaneously with pro- apoptotic BCL-2 family member BAX translocation to mitochondria but before caspases activation [42]. In addition to increased mitochondrial fragmentation, A549 TRAP1depleted cells showed elevated BAX and reduced BCL-xL protein levels. This shift in the balance between pro-apoptotic and pro-survival BCL-2 family proteins was accompanied by an increase in the expression and activity of caspase 3. Surprisingly, caspase 3/7 activation in A549 TRAP1-depleted cells appears to occur in a caspase 8 and/or 9-independent manner suggesting that in these cells, caspase $3 / 7$ undergo activation through alternative pathways. Increased activity of cathepsin B suggests that some degree of lysosomal permeabilization may be occurring. Cytosolic cathepsins may contribute to degradation of BCL-xL [43] and activation of caspase 3 [44]. These results argue that decreased cell viability and increased cell death in A549 TRAP1-depleted cells are due to an apoptotic process.

TRAP1 depletion is associated with activation of cellular stress responses including ER stress protection [11] and mitochondrial UPR in Drosophila [45] and activation of autophagy and the UPR in tumor cells [9]. Since these responses can activate apoptotic pathways we investigated their role in TRAP1-depleted loss of cell viability. Our studies showed loss of $\Delta \Psi_{\mathrm{m}}$, mitochondrial fragmentation and increased expression of DRP1 all of which are associated with accumulation of damaged mitochondria and activation of macroautophagy to remove them $[46,47]$. A549 siTRAP cells showed a marked increase in the overall lysosome content suggesting activation of autophagy which transports cargo to the lysosome for degradation.

Initial studies showed that TRAP1 silencing resulted in a generalized decrease in the expression of enzymes involved in the autophagic pathway, such as BECLIN-1, and proteins that are part of both ATG12 and LC3 ubiquitin-like systems. However, enzyme levels do not reflect autophagic flux [32]. Measurement of autophagic substrate SQSTM1 levels showed 
decreased expression in TRAP1 depleted cells. Further, the accumulation of LC3-II upon inhibition of late phase autophagy with Bafilomycin A supports an increase in the autophagic flux in cells with decreased TRAP1 expression. In addition, increased levels of the lysosomal receptor for chaperone-mediated autophagy (CMA) cargo, LAMP2A, raise the possibility that CMA is also involved. No change in expression of the UPR-related chaperones HSP90, GRP78 and GRP94 was measured suggesting that TRAP1 depletion in these cells may not alter the UPR.

As disturbances in the autophagy flux has been associated with cancer development and progression [48], our results suggest that autophagy in A549 cells constitutes a mechanism to promote cell survival. Additionally, the effect of rapamycin treatment in TRAP1-silenced cells growth reinforces the role of this chaperone in the regulation of survival autophagy. Inhibition of autophagy by treatment with 3-MA leads to decreased cell viability in both TRAP1-expressing and TRAP-depleted cells indicating that autophagy promotes cell viability in these cells. However, treatment with Rapamycin, predicted to activate autophagy by inhibition of mTOR, showed contradictory results. Interestingly, TRAP1-depleted cells proliferation after rapamycin treatment was higher than their basal growth and control cells incubated with the same drug showed similar proliferation rates to that of non-treated siTRAP1 cells. The later observations show that by stimulating autophagy in TRAP1expressing tumor cells it may be possible to mimic the effect of TRAP1 inhibition. These results demonstrate that autophagy at endogenous levels is protective for A549 cells. In contrast, autophagy inhibition does not seem to have a strong effect on MRC-5 cells whereas autophagy induction leads to a higher decrease in overall cell viability.

Altogether these results highlight TRAP1 involvement in the main cellular quality control mechanisms concerning the lysosomal system in the A549 tumor cell line. Autophagy activation upon TRAP1 silencing in these cells seems to have a protective role in maintaining homeostasis upon loss of the mitochondrial chaperone. However, TRAP1 depletion results in mitochondrial damage in tumor cells, consequent accumulation of misfolded proteins and 
damaged organelles, to which cells respond by increasing autophagy levels to avoid apoptosis.

The translation of our in vitro data to clinical application is not straightforward. One current limitation is the lack of in vivo evidence that TRAP1 targeting by pharmacological means inhibits tumour growth. In fact, the effectiveness of targeted therapies for cancers is extremely context dependent. For example, the complexity of the role of autophagy in therapeutic responses to therapy in NSCLC has been demonstrated [49]. Furthermore, recent data demonstrated that cytosolic Hsp90alpha and TRAP1 modulate growth and lung metastasis in vivo, although they do not play any role in the initiation of breast cancer. It remains to be demonstrated whether the same occurs in lung cancer and whether modulation of autophagy plays a role [50].

In conclusion, our study provides evidence that modulation of TRAP1 expression influences the effect of autophagy on A549 cell viability providing new insights to possible therapeutic approaches regarding TRAP1 overexpressing tumors as the described results support the hypothesis that autophagy inhibition could constitute a good approach to induce tumor cell death with no strong implications to normal cells.

\section{ACKNOWLEDGEMENTS}

Sponsored by the European Regional Development Fund (ERDF) through the COMPETE 2020 - Operational Programme for Competitiveness and Internationalisation and Portuguese national funds via FCT-Fundação para a Ciência e a Tecnologia, projects POCI-01-0145FEDER-016390:CANCEL STEM, and IF/01316/2014. Also supported by POCI-01-0145FEDER-007440, and PCTI project (Government of the Principality of Asturias and ERDF) with reference GRUPIN14-071. The funding agencies had no role in study design, experimentation, or writing the manuscript. The authors have no conflict of interests. 


\section{AUTHOR CONTRIBUTIONS}

IAB, IVN, RL, AFB, RG performed the research work; IAB, IVN, PMS, PJO wrote the manuscript; IAB prepared the figures; PJO, IVN and PMS provided supervision of the work; all authors critically read the manuscript and approved its submission. 


\section{REFERENCES}

1 Koh H and Chung J. PINK1 as a molecular checkpoint in the maintenance of mitochondrial function and integrity. Mol Cells 2012;34:7-13.

2 Landriscina M, Laudiero G, Maddalena F, Amoroso MR, Piscazzi A, Cozzolino F et al. Mitochondrial chaperone Trap1 and the calcium binding protein Sorcin interact and protect cells against apoptosis induced by antiblastic agents. Cancer Res 2010;70:6577-86.

3 Xiang F, Huang YS, Shi XH and Zhang Q. Mitochondrial chaperone tumour necrosis factor receptor-associated protein 1 protects cardiomyocytes from hypoxic injury by regulating mitochondrial permeability transition pore opening. FEBS J 2010;277:1929-38.

4 Zhang Y, Jiang DS, Yan L, Cheng KJ, Bian ZY and Lin GS. HSP75 protects against cardiac hypertrophy and fibrosis. J Cell Biochem 2011;112:1787-94.

5 Montesano Gesualdi N, Chirico G, Pirozzi G, Costantino E, Landriscina M and Esposito F. Tumor necrosis factor-associated protein 1 (TRAP-1) protects cells from oxidative stress and apoptosis. Stress 2007;10:342-50.

6 Hua G, Zhang Q and Fan Z. Heat shock protein 75 (TRAP1) antagonizes reactive oxygen species generation and protects cells from granzyme Mmediated apoptosis. J Biol Chem 2007;282:20553-60.

7 Im CN, Lee JS, Zheng Y and Seo JS. Iron chelation study in a normal human hepatocyte cell line suggests that tumor necrosis factor receptor-associated protein 1 (TRAP1) regulates production of reactive oxygen species. $J$ Cell Biochem 2007;100:474-86. 
8 Kang BH, Plescia J, Dohi T, Rosa J, Doxsey SJ and Altieri DC. Regulation of tumor cell mitochondrial homeostasis by an organelle-specific Hsp90 chaperone network. Cell 2007;131:257-70.

9 Amoroso MR, Matassa DS, Laudiero G, Egorova AV, Polishchuk RS, Maddalena F et al. TRAP1 and the proteasome regulatory particle TBP7/Rpt3 interact in the endoplasmic reticulum and control cellular ubiquitination of specific mitochondrial proteins. Cell Death Differ 2012;19:592-604.

10 Siegelin MD, Dohi T, Raskett CM, Orlowski GM, Powers CM, Gilbert CA et al. Exploiting the mitochondrial unfolded protein response for cancer therapy in mice and human cells. J Clin Invest 2011;121:1349-60.

11 Takemoto K, Miyata S, Takamura H, Katayama T and Tohyama M. Mitochondrial TRAP1 regulates the unfolded protein response in the endoplasmic reticulum. Neurochem Int 2011;58:880-7.

12 Caino MC, Chae YC, Vaira V, Ferrero S, Nosotti M, Martin NM et al. Metabolic stress regulates cytoskeletal dynamics and metastasis of cancer cells. J Clin Invest 2013;123:2907-20.

13 Chae YC, Caino MC, Lisanti S, Ghosh JC, Dohi T, Danial NN et al. Control of tumor bioenergetics and survival stress signaling by mitochondrial HSP90s. Cancer Cell 2012;22:331-44.

14 Masuda Y, Shima G, Aiuchi T, Horie M, Hori K, Nakajo S et al. Involvement of tumor necrosis factor receptor-associated protein 1 (TRAP1) in apoptosis induced by beta-hydroxyisovalerylshikonin. J Biol Chem 2004;279:42503-15.

15 Costantino E, Maddalena F, Calise S, Piscazzi A, Tirino V, Fersini A et al. TRAP1, a novel mitochondrial chaperone responsible for multi-drug 
resistance and protection from apoptotis in human colorectal carcinoma cells. Cancer Lett 2009;279:39-46.

16 Fang W, Li X, Jiang Q, Liu Z, Yang H, Wang S et al. Transcriptional patterns, biomarkers and pathways characterizing nasopharyngeal carcinoma of Southern China. J Transl Med 2008;6:32.

17 Leav I, Plescia J, Goel HL, Li J, Jiang Z, Cohen RJ et al. Cytoprotective mitochondrial chaperone TRAP-1 as a novel molecular target in localized and metastatic prostate cancer. Am J Pathol 2010;176:393-401.

18 Siegelin MD. Inhibition of the mitochondrial Hsp90 chaperone network: a novel, efficient treatment strategy for cancer? Cancer Lett 2013;333:133-46.

19 Cerami E, Gao J, Dogrusoz U, Gross BE, Sumer SO, Aksoy BA et al. The cBio cancer genomics portal: an open platform for exploring multidimensional cancer genomics data. Cancer Discov 2012;2:401-4.

20 Gao J, Aksoy BA, Dogrusoz U, Dresdner G, Gross B, Sumer SO et al. Integrative analysis of complex cancer genomics and clinical profiles using the cBioPortal. Sci Signal 2013;6:pl1.

21 Agorreta J, Hu J, Liu D, Delia D, Turley H, Ferguson DJ et al. TRAP1 regulates proliferation, mitochondrial function, and has prognostic significance in NSCLC. Mol Cancer Res 2014;12:660-9.

22 Skehan P, Storeng R, Scudiero D, Monks A, McMahon J, Vistica D et al. New colorimetric cytotoxicity assay for anticancer-drug screening. J Natl Cancer Inst 1990;82:1107-12.

23 Petronilli V, Miotto G, Canton M, Brini M, Colonna R, Bernardi P et al. Transient and long-lasting openings of the mitochondrial permeability 
transition pore can be monitored directly in intact cells by changes in mitochondrial calcein fluorescence. Biophys $J$ 1999;76:725-34.

24 Tian X, Ma P, Sui CG, Meng FD, Li Y, Fu LY et al. Suppression of tumor necrosis factor receptor-associated protein 1 expression induces inhibition of cell proliferation and tumor growth in human esophageal cancer cells. FEBS J 2014;281:2805-19.

25 Felts SJ, Owen BA, Nguyen P, Trepel J, Donner DB and Toft DO. The hsp90related protein TRAP1 is a mitochondrial protein with distinct functional properties. J Biol Chem 2000;275:3305-12.

26 Cechetto JD and Gupta RS. Immunoelectron microscopy provides evidence that tumor necrosis factor receptor-associated protein 1 (TRAP-1) is a mitochondrial protein which also localizes at specific extramitochondrial sites. Exp Cell Res 2000;260:30-9.

27 Dagda RK, Cherra SJ, 3rd, Kulich SM, Tandon A, Park D and Chu CT. Loss of PINK1 function promotes mitophagy through effects on oxidative stress and mitochondrial fission. $J$ Biol Chem 2009;284:13843-55.

28 Bojes HK, Suresh PK, Mills EM, Spitz DR, Sim JE and Kehrer JP. Bcl-2 and Bcl-xL in peroxide-resistant A549 and U87MG cells. Toxicol Sci 1998;42:109-16.

29 Ben-Ari Z, Mor E, Azarov D, Sulkes J, Tor R, Cheporko Y et al. Cathepsin B inactivation attenuates the apoptotic injury induced by ischemia/reperfusion of mouse liver. Apoptosis 2005;10:1261-9.

30 Johansson AC, Steen H, Ollinger K and Roberg K. Cathepsin D mediates cytochrome c release and caspase activation in human fibroblast apoptosis induced by staurosporine. Cell Death Differ 2003;10:1253-9. 
31 Zheng X, Chu F, Mirkin BL, Sudha T, Mousa SA and Rebbaa A. Role of the proteolytic hierarchy between cathepsin L, cathepsin D and caspase-3 in regulation of cellular susceptibility to apoptosis and autophagy. Biochim Biophys Acta 2008;1783:2294-300.

32 Klionsky DJ, Abdelmohsen K, Abe A, Abedin MJ, Abeliovich H, Acevedo Arozena A et al. Guidelines for the use and interpretation of assays for monitoring autophagy (3rd edition). Autophagy 2016;12:1-222.

33 Yang YP, Hu LF, Zheng HF, Mao CJ, Hu WD, Xiong KP et al. Application and interpretation of current autophagy inhibitors and activators. Acta Pharmacol Sin 2013;34:625-35.

34 Bjorkoy G, Lamark T, Pankiv S, Overvatn A, Brech A and Johansen T. Monitoring autophagic degradation of p62/SQSTM1. Methods Enzymol 2009;452:181-97.

35 Kaushik S and Cuervo AM. Chaperone-mediated autophagy: a unique way to enter the lysosome world. Trends Cell Biol 2012;22:407-17.

36 Gozuacik D and Kimchi A. Autophagy as a cell death and tumor suppressor mechanism. Oncogene 2004;23:2891-906.

37 Ogier-Denis E and Codogno P. Autophagy: a barrier or an adaptive response to cancer. Biochim Biophys Acta 2003;1603:113-28.

38 Basit F, van Oppen LM, Schockel L, Bossenbroek HM, van Emst-de Vries SE, Hermeling JC et al. Mitochondrial complex I inhibition triggers a mitophagy-dependent ROS increase leading to necroptosis and ferroptosis in melanoma cells. Cell Death Dis 2017;8:e2716.

39 Youle RJ and van der Bliek AM. Mitochondrial fission, fusion, and stress. Science 2012;337:1062-5. 
40 Butler EK, Voigt A, Lutz AK, Toegel JP, Gerhardt E, Karsten P et al. The mitochondrial chaperone protein TRAP1 mitigates alpha-Synuclein toxicity. PLoS Genet 2012;8:e1002488.

41 Takamura H, Koyama Y, Matsuzaki S, Yamada K, Hattori T, Miyata S et al. TRAP1 controls mitochondrial fusion/fission balance through Drp1 and Mff expression. PLoS One 2012;7:e51912.

42 Youle RJ and Karbowski M. Mitochondrial fission in apoptosis. Nat Rev Mol Cell Biol 2005;6:657-63.

43 de Castro MAG, Bunt G and Wouters FS. Cathepsin B launches an apoptotic exit effort upon cell death-associated disruption of lysosomes. Cell Death Discovery 2016;2:16012.

44 Johansson AC, Appelqvist H, Nilsson C, Kagedal K, Roberg K and Ollinger K. Regulation of apoptosis-associated lysosomal membrane permeabilization. Apoptosis 2010;15:527-40.

45 Morrow G, Kim HJ, Pellerito O, Bourrelle-Langlois M, Le Pecheur M, Groebe K et al. Changes in Drosophila mitochondrial proteins following chaperone-mediated lifespan extension confirm a role of Hsp22 in mitochondrial UPR and reveal a mitochondrial localization for cathepsin D. Mech Ageing Dev 2016;155:36-47.

46 Twig G, Elorza A, Molina AJ, Mohamed H, Wikstrom JD, Walzer G et al. Fission and selective fusion govern mitochondrial segregation and elimination by autophagy. EMBO J 2008;27:433-46.

47 Rodriguez-Enriquez S, He L and Lemasters JJ. Role of mitochondrial permeability transition pores in mitochondrial autophagy. Int J Biochem Cell Biol 2004;36:2463-72. 
48 Levine B and Kroemer G. Autophagy in the pathogenesis of disease. Cell 2008;132:27-42.

49 Saleh T, Cuttino L and Gewirtz DA. Autophagy is not uniformly cytoprotective: a personalized medicine approach for autophagy inhibition as a therapeutic strategy in non-small cell lung cancer. Biochim Biophys Acta 2016.

50 Vartholomaiou E, Madon-Simon M, Hagmann S, Muhlebach G, Wurst W, Floss T et al. Cytosolic Hsp90alpha and its mitochondrial isoform Trap1 are differentially required in a breast cancer model. Oncotarget 2017;8:17428-42. 


\section{FIGURE LEGENDS}

Figure 1. TRAP1 silencing results in mitochondrial dysfunction. (A) Confocal images illustrate siTRAP1 and siCTL cells labeled with anti-TOM20 and anti-TRAP1 antibodies to determine TOM20 and TRAP1 co-localization. DAPI labels nuclei. Scale bar $=8 \mu \mathrm{m}$. (B) ATP, ADP and AMP measurements taken by HPLC indicate a decreased ATP/ADP ratio and energy charge in siTRAP1 cells. (C) Basal oxygen consumption measured with a Seahorse $\mathrm{XF}^{\mathrm{e}} 96$ Extracellular Flux Analyzer and displayed as oxygen consumption rate (OCR) is higher in siCTL than in siTRAP1 cells. Data are means \pm SEM from $n=3$ experiments. (D) Measurement of oxygen consumption rate (OCR) in both types of A549 cells using Seahorse Biosciences assay plates shows decreased respiration in siTRAP1 cells. Graphs are representative of three independent experiments and show the effects of mitochondrial inhibitors, (a) Oligomycin (2.5 $\mu \mathrm{M})$, (b) and (c) FCCP (500 nM) and (d) antimycin A (2.5 $\mu \mathrm{M})$ plus rotenone $(2.5 \mu \mathrm{M})$ injected as indicated. (E) Images of MitoSOX fluorescence in siTRAP1 and siCTL cells show an increase in mitochondrial superoxide anion production in siTRAP1 cells. Scale bar $=8 \mu \mathrm{m}$. (F) TMRM fluorescence measured by flow cytometry reveals a lower mitochondrial membrane potential in siTRAP1 cells. Data are shown as relative fluorescence units (RFU) that represent the mean average of geometric mean values \pm SEM from three independent experiments. (G) Flow cytometric measurement of cobalt quenching of calcein fluorescence as an end-point for mPTP opening reveal a more closed conformation in siTRAP1 than in siCTL cells. Cyclosporin A $(2 \mu \mathrm{M})$ was able to close the pore in both types of A549 cells. Data are shown as relative fluorescence units (RFU) that represent the mean average of geometric mean values \pm SEM, normalized to fluorescence of cells incubated with calcein only for $\mathrm{n}=3 .{ }^{*} \mathrm{p}<0.05 ; * * \mathrm{p}<0.01 ; * * * \mathrm{p}<0.001$ vs. siCTL cells.

Figure 2. TRAP1 silencing results in mitochondrial fragmentation. (A) Confocal images obtained using MitoTracker Red (MTR) for mitochondrial labeling and DAPI for nuclear labeling show alterations in mitochondrial morphology between siCTL and siTRAP1 A549 
cells. Bar charts shows mitochondrial elongation and interconnectivity measured using Image $\mathrm{J}$ in fluorescent images of siCTL and siTRAP1 cells stained with MTR. Data show mean \pm SEM from three separate experiments ( 15 cells per experimental condition and experiment). (B) Levels of proteins involved in mitochondrial dynamics (DRP1, FIS1, MFN1 and OPA1) indicate the occurrence of mitochondrial fission in siTRAP1 cells. Bar charts show means of optical density (O.D.) \pm SEM normalized to actin, from at least three separate immunoblots. ${ }^{*} \mathrm{p}<0.05 ; * * * \mathrm{p}<0.001$ vs. siCTL cells.

Figure 3. TRAP1 silencing alters cell death machinery. Expression of proteins involved in cell death (A) BAX, BCL-xL, and (B) caspase-3 indicates alterations in apoptotic pathways in A549 cells transfected with a TRAP1 siRNA (siTRAP1) in comparison with A549 cells transfected with a scrambled siRNA (siCTL). Bar charts show means of optical density (O.D.) \pm SEM normalized to actin, from at least three separate immunoblots. (C) Caspase 3/7-like activity showed an over-activation of caspase 3/7-like activity in siTRAP1 cells. (D) Cathepsin D and B activities were determined to test the activation of lysosomal processes of cell death, showing cathepsin B activation in siTRAP1 cells. Data are means of enzymatic units per mg of protein \pm SEM, from at least five independent experiments. (E) Immunoblot analysis against cathepsin D and cathepsin B shows decreased expression of both proteases upon TRAP1 silencing. (F) Caspases 8-, 9-like activities determined in both types of A549 cells showed no alterations in the initiator caspases. (G) Representative western blots. Data are expressed as means \pm SEM from at least three independent experiments. Bar charts show means of optical density (O.D.) \pm SEM normalized to actin, from at least three separate immunoblots. ${ }^{*} \mathrm{p}<0.05 ; * * \mathrm{p}<0.01 ; * * \mathrm{p}<0.001$ vs. siCTL cells.

Figure 4. TRAP1 silencing activates macroautophagy. (A) LC3 protein expression analysis reveals no alterations in the amount of the marker of autophagosomes LC3-II between both types of A549 cells. To evaluate autophagic flux, both types of cells were treated with 
bafilomycin A (BafA1) showing a higher accumulation of LC3-II in siTRAP1 cells. Bar charts show means of optical density (O.D.) \pm SEM normalized to actin, from at least three separate immunoblots. (B) Immunoblot analysis against the autophagy-related proteins BECLIN-1, ATG5, ATG12, ATG3 and ATG7 suggests a down-regulation in the autophagic machinery in A549 cells transfected with a TRAP1 siRNA (siTRAP1) in comparison with A549 cells transfected with a scrambled siRNA (siCTL). However, p62 expression was lower in siTRAP1 cells indicating that autophagic flux is higher in TRAP1-silenced cells. (C) Labelling of acidic compartments using Lysotracker Green reveals the development of acidic organelles after TRAP1 silencing. Histogram shows means of corrected total cell fluorescence \pm SEM from three independent experiments ( 15 cells per experimental condition and experiment). (D) Protein expression of HCS70 shows no changes after TRAP1 depletion but LAMP-2A receptor shows a higher protein expression in siTRAP1 cells. Bar charts show means of optical density (O.D.) \pm SEM normalized to actin, from at least three separate immunoblots. $* \mathrm{p}<0.05 ; * * \mathrm{p}<0.01 ; * * * \mathrm{p}<0.001$ vs. siCTL cells.

Figure 5. Effect of the autophagic drugs, (A) 3-methyladenine (3-MA, $5 \mathrm{mM}$ ), and (B) rapamycin $(100 \mathrm{nM})$, on cell viability. Rapamycin increased the proliferation of siTRAP1. Data are expressed as percentage of the control (vehicle only) for each time point. Data are means \pm SEM from $n=5$. \# Represents comparison with siTRAP1 cells treated with either rapamycin or 3-MA, * represents comparison with siTRAP1 cells, and • represents comparison with siCTL cells treated either with rapamycin or 3-MA. 


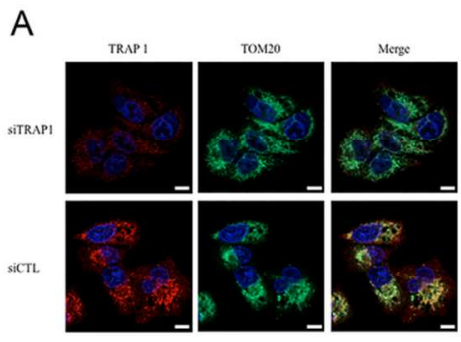

C

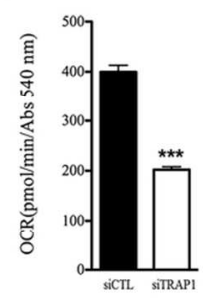

D

B

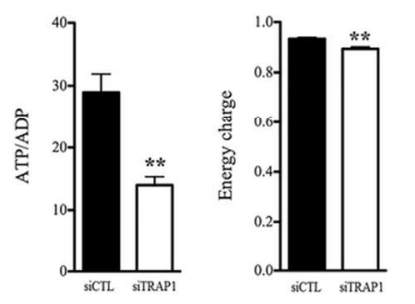

E

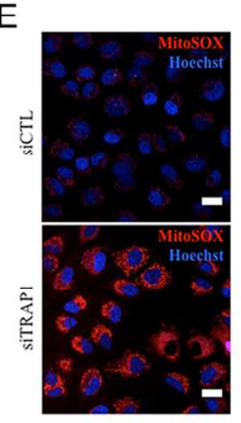

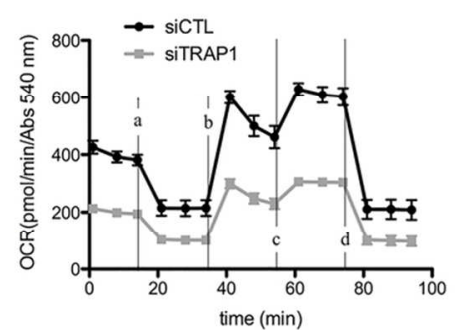

F

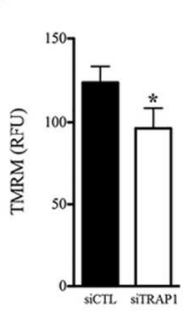

G

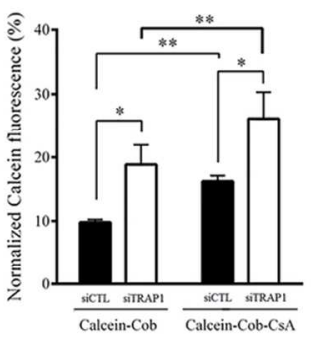

Figure 1. TRAP1 silencing results in mitochondrial dysfunction. (A) Confocal images illustrate siTRAP1 and siCTL cells labeled with anti-TOM20 and anti-TRAP1 antibodies to determine TOM20 and TRAP1 COlocalization. DAPI labels nuclei. Scale bar $=8 \mu \mathrm{m}$. (B) ATP, ADP and AMP measurements taken by HPLC indicate a decreased ATP/ADP ratio and energy charge in siTRAP1 cells. (C) Basal oxygen consumption measured with a Seahorse XFe96 Extracellular Flux Analyzer and displayed as oxygen consumption rate

(OCR) is higher in siCTL than in siTRAP1 cells. Data are means \pm SEM from $n=3$ experiments. (D) Measurement of oxygen consumption rate (OCR) in both types of A549 cells using Seahorse Biosciences assay plates shows decreased respiration in siTRAP1 cells. Graphs are representative of three independent experiments and show the effects of mitochondrial inhibitors, (a) Oligomycin $(2.5 \mu \mathrm{M})$, (b) and (c) FCCP $(500 \mathrm{nM})$ and $(\mathrm{d})$ antimycin $\mathrm{A}(2.5 \mu \mathrm{M})$ plus rotenone $(2.5 \mu \mathrm{M})$ injected as indicated. (E) Images of MitoSOX fluorescence in SITRAP1 and siCTL cells show an increase in mitochondrial superoxide anion production in siTRAP1 cells. Scale bar $=8 \mu \mathrm{m}$. (F) TMRM fluorescence measured by flow cytometry reveals a lower mitochondrial membrane potential in siTRAP1 cells. Data are shown as relative fluorescence units (RFU) that represent the mean average of geometric mean values \pm SEM from three independent experiments. (G) Flow cytometric measurement of cobalt quenching of calcein fluorescence as an end-point for mPTP opening reveal a more closed conformation in siTRAP1 than in siCTL cells. Cyclosporin A ( $2 \mu \mathrm{M})$ was able to close the pore in both types of A549 cells. Data are shown as relative fluorescence units (RFU) that represent the mean average of geometric mean values \pm SEM, normalized to fluorescence of cells incubated with calcein only for $\mathrm{n}=3$. ${ }^{*} \mathrm{p}<0.05 ; * * \mathrm{p}<0.01 ; * * * \mathrm{p}<0.001$ vs. siCTL cells. 
A

B
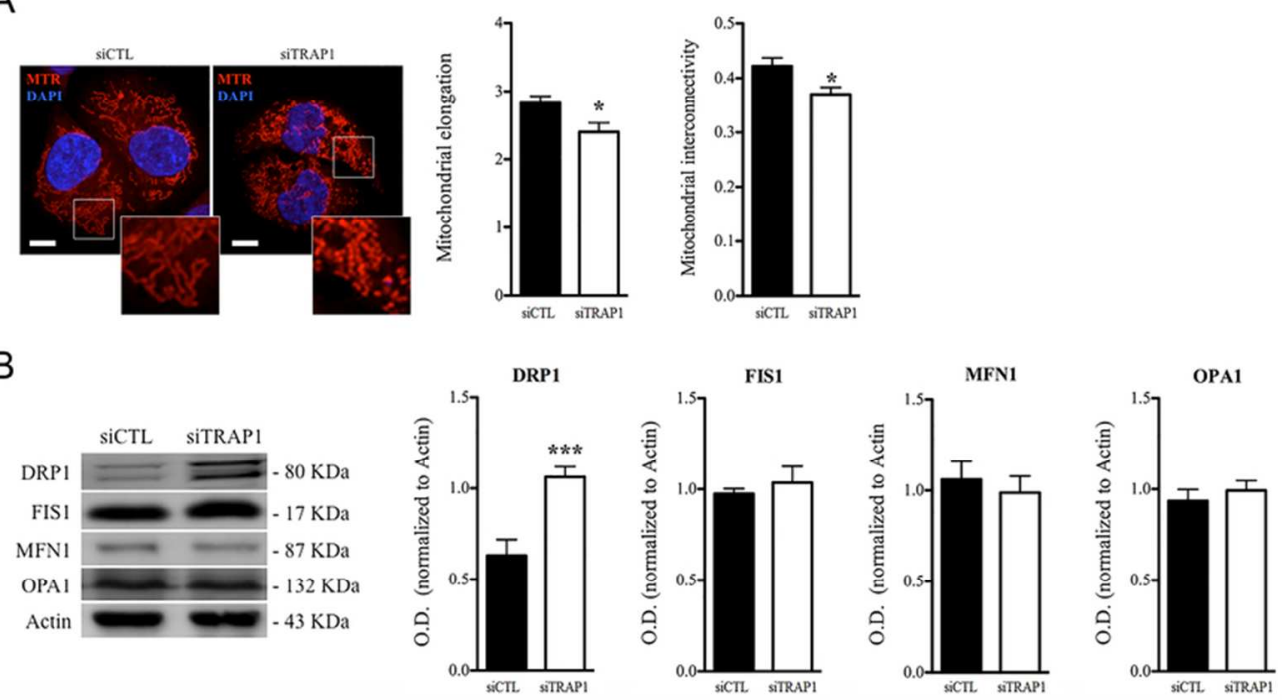

Figure 2 - TRAP1 silencing results in mitochondrial fragmentation. (A) Confocal images obtained using

MitoTracker Red (MTR) for mitochondrial labeling and DAPI for nuclear labeling show alterations in mitochondrial morphology between siCTL and siTRAP1 A549 cells. Bar charts shows mitochondrial elongation and interconnectivity measured using Image $\mathrm{J}$ in fluorescent images of SiCTL and siTRAP1 cells stained with MTR. Data show mean \pm SEM from three separate experiments (15 cells per experimental condition and experiment). (B) Levels of proteins involved in mitochondrial dynamics (DRP1, FIS1, MFN1 and OPA1) indicate the occurrence of mitochondrial fission in siTRAP1 cells. Bar charts show means of optical density (O.D.) \pm SEM normalized to actin, from at least three separate immunoblots. $* p<0.05 ; * * * p<0.001$ vs. siCTL cells. 
A

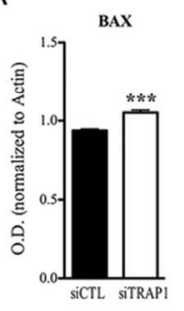

E

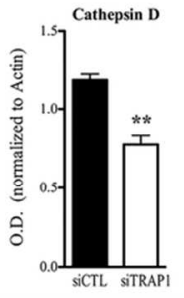

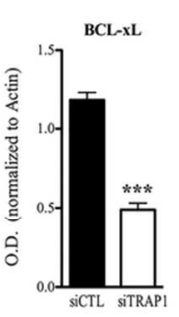

B
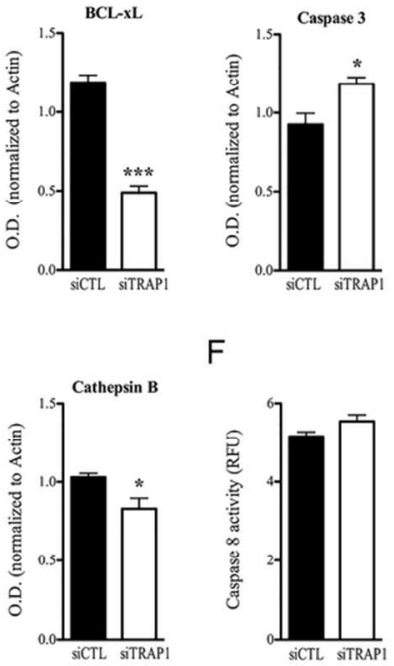

$\mathrm{F}$

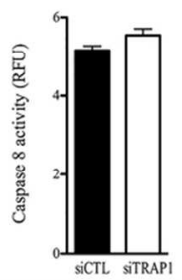

C

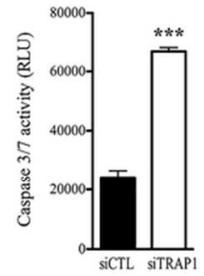

D

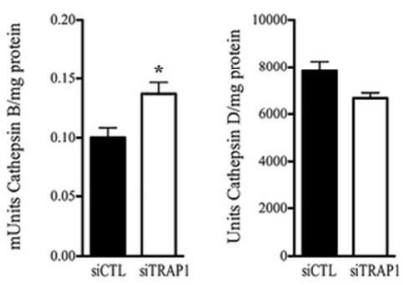

G

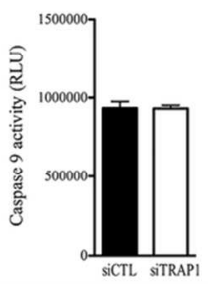

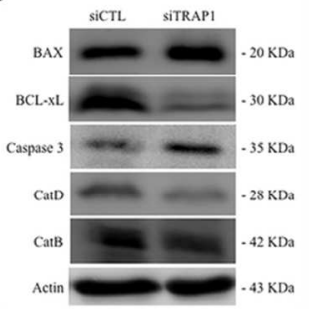

Figure 3. TRAP1 silencing alters cell death machinery. Expression of proteins involved in cell death (A) BAX, $B C L-x L$, and (B) caspase- 3 indicates alterations in apoptotic pathways in A549 cells transfected with a TRAP1 siRNA (siTRAP1) in comparison with A549 cells transfected with a scrambled siRNA (siCTL). Bar charts show means of optical density (O.D.) \pm SEM normalized to actin, from at least three separate immunoblots. (C) Caspase 3/7-like activity showed an over-activation of caspase 3/7-like activity in siTRAP1 cells. (D) Cathepsin D and B activities were determined to test the activation of lysosomal processes of cell death, showing cathepsin B activation in siTRAP1 cells. Data are means of enzymatic units per mg of protein \pm SEM, from at least five independent experiments. (E) Immunoblot analysis against cathepsin $D$ and cathepsin B shows decreased expression of both proteases upon TRAP1 silencing. (F) Caspases 8-, 9-like activities determined in both types of A549 cells showed no alterations in the initiator caspases. (G) Representative western blots. Data are expressed as means \pm SEM from at least three independent experiments. Bar charts show means of optical density (O.D.) \pm SEM normalized to actin, from at least three separate immunoblots. $* p<0.05 ; * * p<0.01 ; * * * p<0.001$ vs. siCTL cells. 
A

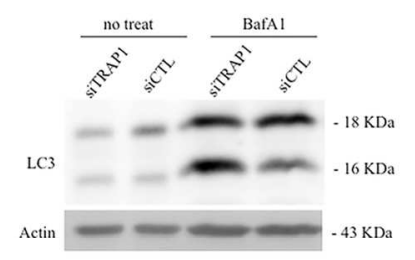

C
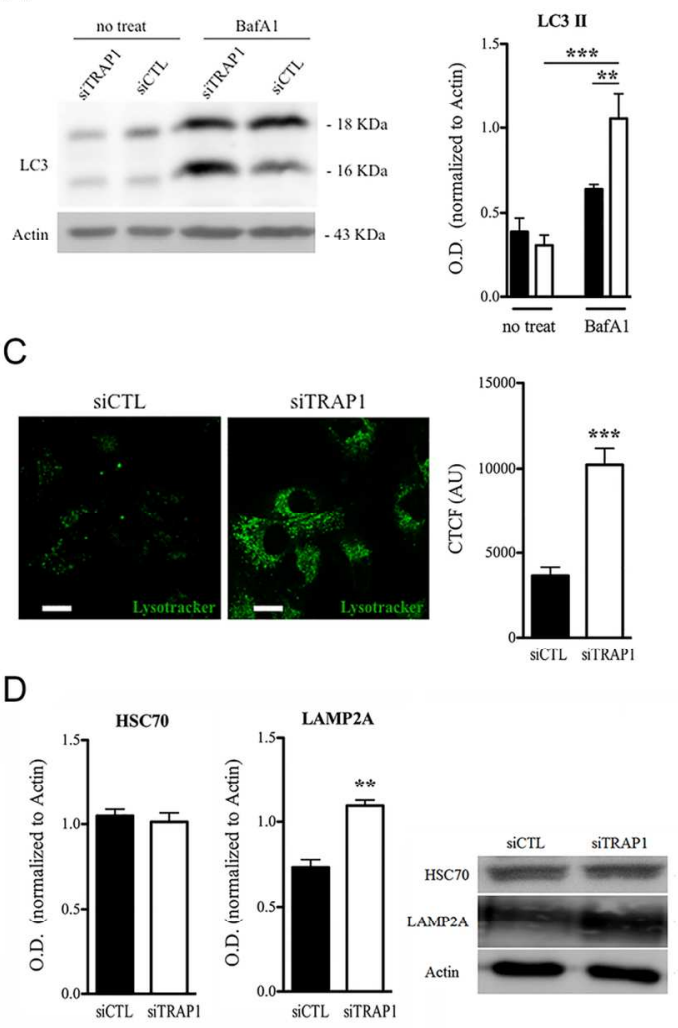

B
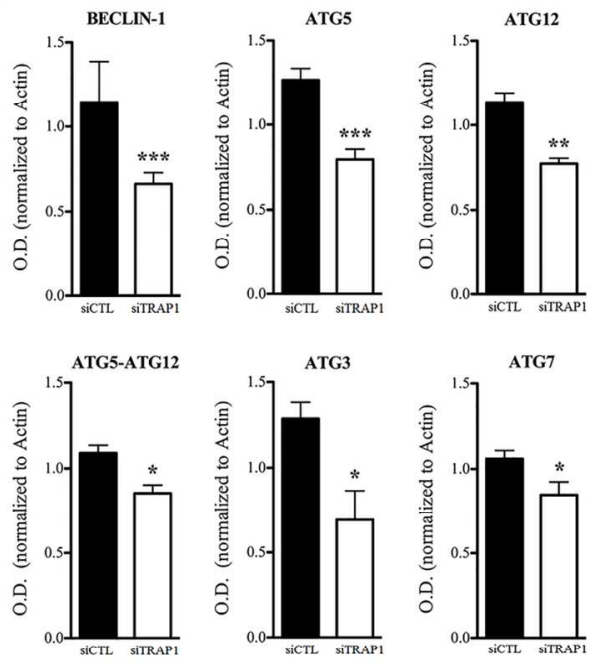

Figure 4. TRAP1 silencing activates macroautophagy. (A) LC3 protein expression analysis reveals no alterations in the amount of the marker of autophagosomes LC3-II between both types of A549 cells. To evaluate autophagic flux, both types of cells were treated with bafilomycin A (BafA1) showing a higher accumulation of LC3-II in siTRAP1 cells. Bar charts show means of optical density (O.D.) \pm SEM normalized to actin, from at least three separate immunoblots. (B) Immunoblot analysis against the autophagy-related proteins BECLIN-1, ATG5, ATG12, ATG3 and ATG7 suggests a down-regulation in the autophagic machinery in A549 cells transfected with a TRAP1 siRNA (siTRAP1) in comparison with A549 cells transfected with a scrambled siRNA (siCTL). However, p62 expression was lower in siTRAP1 cells indicating that autophagic flux is higher in TRAP1-silenced cells. (C) Labelling of acidic compartments using Lysotracker Green reveals the development of acidic organelles after TRAP1 silencing. Histogram shows means of corrected total cell

fluorescence \pm SEM from three independent experiments (15 cells per experimental condition and

experiment). (D) Protein expression of HCS70 shows no changes after TRAP1 depletion but LAMP-2A receptor shows a higher protein expression in siTRAP1 cells. Bar charts show means of optical density (O.D.) \pm SEM normalized to actin, from at least three separate immunoblots. $* \mathrm{p}<0.05 ; * * \mathrm{p}<0.01 ; * * * \mathrm{p}<0.001$ vs. SiCTL cells. 

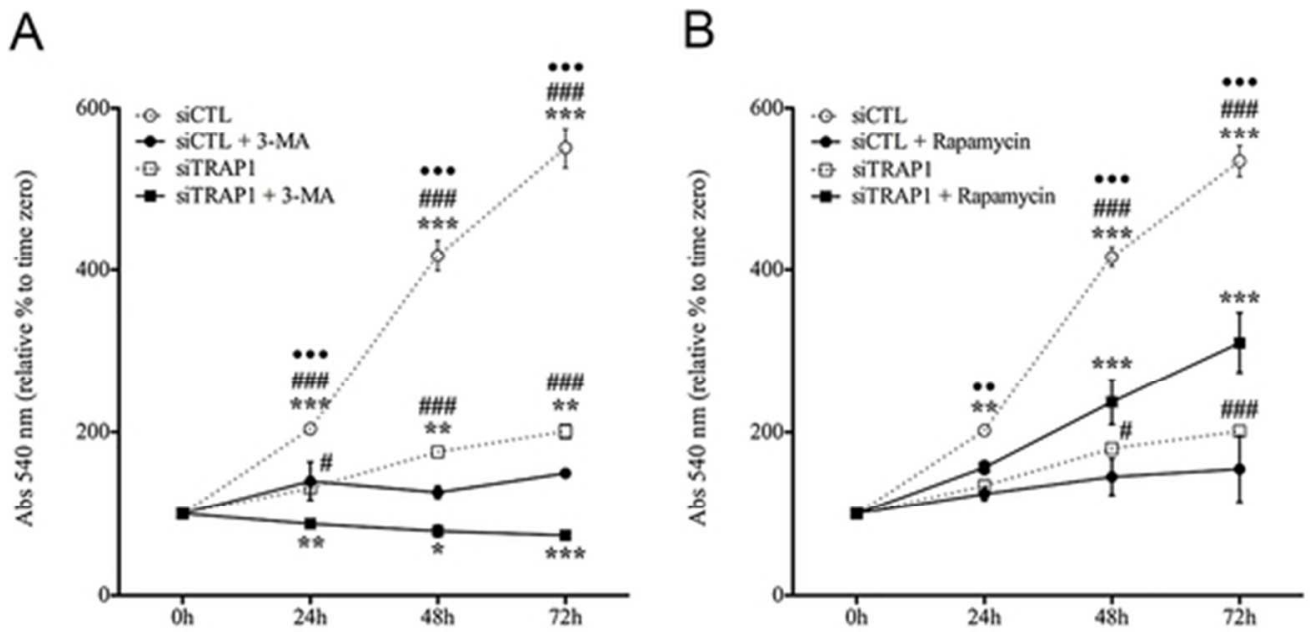

Figure 5. Effect of the autophagic drugs, (A) 3-methyladenine (3-MA, $5 \mathrm{mM})$, and (B) rapamycin (100 nM), on cell viability. Rapamycin increased the proliferation of siTRAP1. Data are expressed as percentage of the control (vehicle only) for each time point. Data are means $\pm S E M$ from $n=5$. \# Represents comparison with siTRAP1 cells treated with either rapamycin or 3-MA, * represents comparison with siTRAP1 cells, and $\square$ represents comparison with siCTL cells treated either with rapamycin or 3-MA.

$46 \times 22 \mathrm{~mm}(300 \times 300 \mathrm{DPI})$ 